\title{
Aprendizajes para la vida a través de la implementación de estrategias didácticas digitales: un estudio comparativo de casos
}

\author{
M. Isabel Pardo Baldoví 1우 ; Diana Marín Suelves ' 10 ; Ana De Castro Calvo ${ }^{2}[0$ \\ ${ }^{1}$ Universitat de València (UV), España; ${ }^{2}$ Florida Universitaria, España
}

Resumen. La sociedad actual se caracteriza por el impacto de las tecnologías en todos los ámbitos de la vida de ciudadanos de diferentes generaciones, etnias, creencias o procedencias. En este contexto, la escuela y sus agentes avanzan hacia la integración de las tecnologías en sus prácticas mediante el uso de diferentes soportes, materiales y estrategias didácticas. El objetivo de este estudio comparativo de casos fue analizar los efectos de la implementación de estrategias didácticas digitales en quinto y sexto de Primaria para conocer el impacto en el aprendizaje de cuestiones clave para la vida. Han participado cuatro centros escolares de dos comunidades autónomas del estado español y la recogida de la información se ha realizado mediante entrevistas semiestructuradas a alumnado y docentes. Los resultados muestran importantes transformaciones propiciadas por las tecnologías, tanto en la dimensión organizativa como en la didáctica. Pero visibilizan carencias para el abordaje de cuestiones de gran calado social, como la igualdad, la diversidad o la participación. Aspectos que trascienden el uso de tecnologías y el desarrollo de la competencia digital, y que destacan la importancia de replantear el papel de las tecnologías en la escuela para alcanzar aprendizajes funcionales para la vida y optimizadores de la sociedad.

Palabras clave: aprendizaje; alumnado; escuela Primaria; tecnología de la educación; transversalidad.

Aprendizagem para a vida através da implementação de estratégias didáticas com o uso de tecnologias digitais: um estudo comparativo de casos

Resumo. A sociedade atual é caracterizada pelo impacto das tecnologias em todas as áreas da vida dos cidadãos de diferentes gerações, etnias, crenças ou origens. Neste contexto, a escola e seus agentes estão caminhando para a integração das tecnologias em suas práticas, através do uso de diferentes suportes, materiais e estratégias didáticas. O objetivo deste estudo comparativo foi analisar os efeitos da implementação de estratégias de ensino com recursos digitais no quinto e sexto ano do Ensino Básico (Fundamental), para conhecer o impacto na aprendizagem de questões fundamentais para a vida. Quatro escolas de duas comunidades autônomas da Espanha participaram e as informações foram coletadas através de entrevistas semiestruturadas com alunos e professores. Os resultados mostram importantes transformações proporcionadas pelas tecnologias digitais, tanto na dimensão organizacional quanto na didática. Contudo, também tornam visíveis carências na abordagem de questões de grande relevância social, tais como igualdade, diversidade ou participação. Aspectos que transcendem o uso de tecnologias e o desenvolvimento da competência digital, e que destacam a importância de repensar o papel das tecnologias na escola para adquirir conhecimentos funcionais para a vida, capazes de melhorar a sociedade.

Palavras-chave: aprendizagem; alunos; ensino básico/fundamental; tecnologia na educação; transversalidade

Life learning through the implementation of digital didactic strategies: a comparative case study Abstract. Today's society is characterized by the impact of technologies in all areas of the daily life of citizens of different generations, ethnic groups, beliefs or backgrounds. In this context, the school and its agents advance towards the integration of technologies in their practices through the use of different supports, materials and teaching strategies. The objective of this comparative case study was to analyze the effects of the implementation of digital teaching strategies to know the impact on learning of key issues for life. Four schools from two autonomous communities in the Spanish state have participated and the information has been collected through semi-structured interviews with students and teachers. The results indicate important transformations fostered by technologies, both in the organizational and didactic sphere. In addition, the research reveals shortcomings for addressing issues of great social significance, such as equality, diversity or participation. These issues go far beyond the use of technology and the development of digital competence, and highlight the importance of rethinking the role of technologies in the school to achieve learnings that are functional for life, moving towards the optimization of society.

Keywords: learning; student; primary school; education technology; transversality. 


\section{Introducción}

En los últimos tiempos, los organismos internacionales han ido estableciendo una serie de metas a alcanzar en los próximos años en base a cuestiones no resueltas y consideradas como prioritarias. Entre estas consideraciones destacan el papel de las tecnologías y la superación de la brecha digital para favorecer el desarrollo integral de la ciudadanía.

Para dar respuesta a estos retos, en el contexto español se han creado distintos planes de actuación estatales. A su vez, la transferencia de competencias en materia educativa a las autonomías, y la consecuente descentralización del sistema escolar, ofrecen un panorama diverso, permitiendo el diseño de programas específicos ajustados a las diferentes realidades, que se implementan en los centros educativos en función de la interpretación realizada y de los medios disponibles para llevarlos a cabo (San Martín, Peirats y Gallardo, 2014), lo que incluye tanto la dotación tecnológica como la competencia digital docente. El reflejo de estas políticas se plasma en los programas fomentados por cada comunidad y en la asunción o no de los proyectos estatales. Esta realidad tiene repercusiones no solo en el desarrollo de políticas autonómicas concretas, sino también en las prácticas educativas llevadas a cabo en los centros, en los procesos de organización y gestión, y en los distintos grados de integración de las tecnologías en las aulas.

Partiendo de este complejo escenario, el presente trabajo consiste en un estudio de casos en cuatro centros escolares de dos comunidades autónomas del Estado español: la Comunitat Valenciana y la Comunidad de Madrid. Con ello, se pretende abordar la contribución de la tecnología educativa respecto al logro de los fines de la educación, analizando su impacto en el proceso de enseñanza-aprendizaje, mediante las estrategias didácticas digitales.

Aunque los contextos son muy distintos, las políticas TIC desarrolladas en las dos comunidades en las que se encuentran los casos analizados presentan similitudes significativas en este ámbito. En común tuvieron su posicionamiento frente a la iniciativa estatal Escuela 2.0 (2009-2012). El rechazo a incorporar este plan y el impulso, en paralelo, de planes propios para la dotación tecnológica de los centros supuso la ausencia de sustento económico por parte del Ministerio de Educación, además de la puesta en práctica de medidas diferenciadas con otras comunidades.

Centrándonos en la singularidad de cada comunidad, a continuación, se describen sus rasgos distintivos respecto a las políticas TIC implementadas en los últimos años. 
La Generalitat Valenciana impulsó la creación de la plataforma institucional Mestre a casa y del sistema operativo Lliurex. En paralelo al desarrollo de Escuela 2.0, se implantó el programa Centros Educativos Inteligentes (CEI) en un reducido número de escuelas que participaron de manera experimental, ya que no se extendió como consecuencia de los recortes presupuestarios. Posteriormente, se desarrollaron el Plan Estratégico de Innovación y Mejora de la Administración de la Generalitat (2014-2016) y la Agenda Digital de la Comunitat Valenciana. En los últimos años, se está fomentando el uso de libros de texto digitales en tabletas, empezando por los cursos superiores de Educación Primaria, para lo que se ha puesto en marcha el programa Xarxa Llibres. Por último, destaca el uso generalizado de plataformas para la gestión y organización, tanto del centro como de las aulas, entre las que destaca ITACA.

En el caso de la Comunidad de Madrid se inició con carácter experimental, en el curso 2010-2011, el proyecto de Institutos de Innovación Tecnológica. Una propuesta alternativa al modelo 1 a 1 de Escuela 2.0, en el que participaron 15 institutos públicos de Educación Secundaria Obligatoria y, en algunos de los casos, el alumnado y profesorado de quinto y sexto de Educación Primaria. Este proyecto tuvo como objetivo principal mejorar el rendimiento del alumnado, tal y como se refleja en la convocatoria de selección de centros en la que se establece que la finalidad del mismo es "[...] contribuir a la mejora de los resultados académicos de los escolares madrileños" (CAM, 2010, p. 51) a través de la utilización de las tecnologías de la Información y de la Comunicación como herramienta de aprendizaje.

En ambos casos, las políticas desarrolladas se han centrado en la promoción de las tecnologías en la enseñanza, la formación del profesorado, la adquisición de habilidades tecnológicas o la utilización de materiales digitales. Pero es mucho más lo que se pretende, ya que, en este contexto, la tecnología se convierte en la herramienta que ha de posibilitar el desarrollo integral de los ciudadanos, que es el fin último de la educación.

Al hilo de la cuestión, abunda la literatura reciente respecto a la incidencia de las tecnologías en la enseñanza (Colás, De Pablos y Ballesta, 2018), y sobre el grado de integración de la tecnología en los centros educativos (Fernández, Fernández y Rodríguez, 2018; Martínez-Serrano, 2019). Asimismo, el uso de materiales didácticos digitales (Area y Rodríguez, 2017; Vidal, Vega y López, 2019) y las percepciones de los diferentes agentes educativos respecto al uso de tecnología en las aulas también han sido focos de interés creciente en la literatura académica (Conde, Ávila, Núñez y Mirabent, 2015; Gallardo, San Nicolás y Cores, 2019; Peirats, Gallardo y San Martín, 2015; Rego-Agraso y Marín, 2019; Sánchez-Antolín y Blanco, 2016). Sin dejar de lado el estudio de las estrategias didácticas digitales (Pardo, San 
Martín y Cuervo, 2019), el aprendizaje mediado por tecnología (Saladino, Marín y San Martín, 2019) o las bondades de la tecnología para aumentar el rendimiento académico (Martín y Mayo, 2019), la motivación (Amores y De Casas, 2019) o mejorar la atención a la diversidad y la inclusión (Cabero y Valencia, 2019), entre otras cuestiones.

Pero, en contraposición a la cantidad de investigaciones recientes mencionadas, respecto a los aprendizajes para la vida, el número de estudios previos es más bien reducido, destacando trabajos como el de Castro, Marín y Sáiz (2019) en el que se analiza el impacto de las tecnologías en la inclusión desde la perspectiva de los diferentes agentes educativos, el de Buxarrais y Ovide (2011), en el que se describe el papel de las herramientas digitales en la educación en valores, o el de Sánchez, Ortega y Vall-Llovera (2012) en el que abordan los factores para la ruptura de la brecha digital de género.

Esta situación ofrece un interesante escenario de partida, ya que si bien los citados estudios evidencian que las tecnologías están provocando la transformación global de la institución escolar y de los roles y tareas asumidas por sus agentes (Pardo y San Martín, 2020); no resulta tan evidente que la naturaleza y orientación de estos cambios repercuta directamente en

20 la optimización social, pese a que en múltiples ocasiones esta afirmación se asume como universal absoluto en el discurso pedagógico (Silva, Rodrigues y Sampayo, 2020).

En base a ello nos preguntamos: ¿con qué finalidades incorpora la escuela las tecnologías digitales en su quehacer cotidiano?, ¿qué usos y valores asocian docentes y discentes al trabajo con herramientas digitales? o, más incisivamente: ¿el trabajo digital en la escuela constituye una innovación que propicia la optimización social o, por el contrario, es un cambio de formato que perpetúa viejas lógicas discursivamente modernizadas? Para tratar de dar respuesta a estos interrogantes se realizó este estudio cuyos objetivos fueron: profundizar en la realidad de la digitalización escolar, para analizar sus implicaciones organizativas y didácticas y, a partir de ellas, poder valorar en qué sentido las tecnologías contribuyen a la transformación de los aprendizajes que el alumnado está adquiriendo y que el profesorado promueve, en base a distintos aspectos claves para la vida en la sociedad del siglo XXI. 


\section{Método}

\subsection{Muestra}

Este estudio comparativo de casos se centra en el análisis de cuatro centros escolares, dos ubicados en la Comunitat Valenciana y dos en la Comunidad de Madrid. Concretamente, el trabajo de campo se ha centrado en las aulas de $5^{\circ}$ y $6^{\circ}$ de Primaria.

El Caso 1 (CPV) es un centro público situado en una zona rural de la provincia de Valencia. Concretamente, es un Centro Rural Agrupado que escolariza un total de 167 alumnos de las etapas de Educación Infantil y Primaria.

El Caso 2 (CCV) es un centro concertado situado en el entorno urbano de la ciudad de Valencia. Cuenta con un total de 368 alumnos escolarizados en las etapas de Educación Infantil, Primaria y Secundaria Obligatoria.

El Caso 3 (CPM) es un gran centro público situado en Algete (Madrid). En esta escuela se escolarizan un total de 604 alumnos entre las etapas de Educación Infantil, Primaria y Secundaria Obligatoria.

Por último, el Caso 4 (CCM) es un centro privado constituido en régimen de cooperativa, ubicado en la localidad de Alcobendas (Madrid). Esta escuela escolariza a un millar de alumnos de edades comprendidas entre los 2 y los 18 años, es decir, desde Educación Infantil hasta Bachillerato.

Los cuatro centros ofrecen realidades distintas, por lo que respecta a su tipología, titularidad, ubicación y alumnado al que atienden. La investigación, cualitativa y de carácter etnográfico, trata de comprender cómo gestionan el trabajo con tecnologías, para generar un conocimiento situado que arroje luz sobre la situación.

\subsection{Instrumento}

Partiendo de los objetivos y del carácter de la investigación, se optó por la entrevista semiestructurada en profundidad como instrumento de recogida de información, al permitir desvelar las visiones y concepciones de los implicados y profundizar en la realidad (Patton, 2002). Se realizaron entrevistas grupales al alumnado de cada clase, con participantes voluntarios o seleccionados por los tutores, en las que se incidía en el impacto generado por las tecnologías en el aula y sobre el proceso de aprendizaje. Paralelamente, 
se realizaron entrevistas individuales a todos los tutores de quinto y sexto de Primaria para conocer los cambios propiciados por las tecnologías sobre el trabajo pedagógico, tanto a nivel organizativo como didáctico.

\subsection{Procedimiento y análisis}

Este estudio estuvo dividido en cinco fases:

- La primera de ellas, consistió en la selección deliberada de los centros participantes, atendiendo a aspectos como el grado de integración de las tecnologías, el interés por participar en la investigación y el contemplar diferentes tipologías de centros educativos. A partir de esta información se cumplimentaron fichas de datos de los centros sobre equipamiento tecnológico, proyectos relacionados con las tecnologías y uso de materiales y estrategias didácticas digitales que sirvieron para contextualizar la investigación.

- La segunda fase fue la recogida de información, mediante la realización de las entrevistas, tras contar con el consentimiento informado.

- La tercera fase consistió en la transcripción de entrevistas y su entrega a los participantes para que confirmaran que quedaba fielmente recogido aquello que querían manifestar.

- La cuarta fase consistió en una reunión de expertos formada por ocho profesionales del ámbito educativo con experiencia en proyectos de investigación sobre tecnología educativa, con la finalidad de identificar aprendizajes considerados como fundamentales para la vida en la sociedad actual, y poder alcanzar el último objetivo, relativo a valorar en qué sentido las tecnologías contribuyen a la promoción de aprendizajes transformadores.

- Finalmente, en la quinta fase se utilizaron matrices elaboradas ad hoc para el análisis de la información, en las que quedó recogida la información con citas textuales de los participantes y la interpretación de las investigadoras en base a distintos aspectos contemplados en las entrevistas y emergidos en la reunión de expertos que se agruparon en tres dimensiones representadas en la figura 1. 


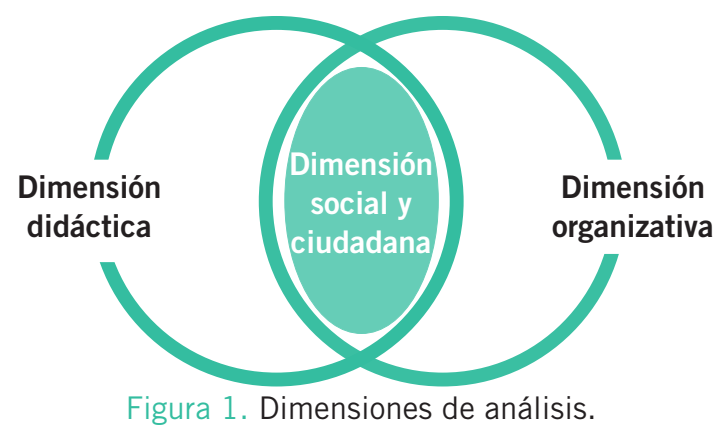

En la figura 1 se reflejan las dimensiones de la escuela a partir de las cuales se vertebra una educación encaminada hacia la transformación social: la dimensión didáctica y la organizativa, como cimientos básicos de la escuela a partir de los cuales se gestiona y desarrolla la tarea educativa, que contemplan la dimensión social y ciudadana, representada como dimensión transversal en la que confluyen, y hacia la cual se dirigen, las otras dos.

De cada una de estas dimensiones emergen una serie de categorías relacionadas. La dimensión organizativa alude tanto a aspectos de relación entre los agentes de la escuela, como a la gestión de la vida en el aula y el centro, el contexto escolar, o el plan de acción de la escuela. En cuanto a la dimensión didáctica se contemplan categorías como las estrategias didácticas y la metodología, el aprendizaje y la formación o la innovación educativa. Finalmente, en relación a la dimensión social y ciudadana se contemplan elementos necesarios para la mejora de la sociedad y del proceso educativo transformador tales como la atención a la diversidad, la perspectiva de género, la igualdad de oportunidades, la sostenibilidad o la participación desde una perspectiva extendida.

\section{Resultados}

En la actual sociedad digital, la escuela cambia progresivamente sus formatos; los procesos de renovación e innovación que se producen en su seno se vinculan con la introducción de herramientas digitales, encaminadas a ofrecer una educación actualizada, tal y como expone una de las docentes entrevistadas:

Yo creo que el maestro debe ir cambiando acorde a los cambios de la sociedad, y si la sociedad ha cambiado con las nuevas tecnologías pues entonces tú te tienes que adaptar a esos cambios tecnológicos. [...] porque 
estamos en la era tecnológica y virtual, y tenemos que darlo en el colegio para no tener problemas, y enseñarlo a esos alumnos a vivir en la sociedad (CPV_ET5, I. 293-300).

La visión de la docente constituye una tendencia generalizada entre los agentes escolares de los centros estudiados, quienes, salvo reducidas excepciones, manifiestan una visión positiva de las tecnologías digitales, considerándolas elementos imprescindibles en la educación actual. Este posicionamiento es auspiciado desde distintos sectores y agencias sociales, entre los que destacan los gobiernos estatales y autonómicos (como se ha destacado anteriormente), así como organismos supranacionales, entidades privadas y buena parte de la literatura académica actual.

Partiendo de estos posicionamientos, en las últimas décadas asistimos a una expansión masiva de las tecnologías digitales en la escuela y a la proliferación de un discurso que las vincula directamente con la optimización educativa y social, que provoca la irrupción de nuevas formas de pensar y hacer educación, de concebir y abordar el currículum y la escuela, mediatizadas por la lógica digital (Loveless y Williamson, 2017). Este fenómeno está provocando una redefinición de la escuela en sentido holístico, tanto por lo que respecta a la dimensión organizativa como a la didáctica, e incluso a la orientación y sentido otorgado al proceso educativo. Aspectos que pueden observarse en la Figura 2, que muestra los conceptos más citados por los entrevistados en relación al trabajo digital en la escuela, donde se destacan aspectos relacionados con múltiples fenómenos y procesos, como el uso de materiales y artefactos, la gestión y organización de tareas y procesos, las relaciones entre los agentes, entre otros.

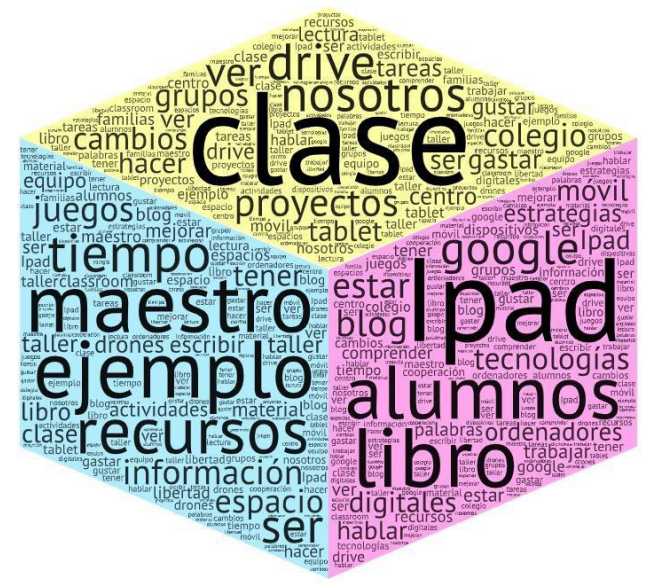

Figura 2. Conceptos más citados por los entrevistados en relación al trabajo digital en la escuela. 
La introducción de tecnologías digitales en la escuela es un fenómeno de gran complejidad, que implica la conexión entre distintos agentes e instancias, y que remite a aspectos muy diversos. En consecuencia, tratar de analizar y comprender la realidad resulta una tarea compleja.

A continuación, se presentan los resultados obtenidos en cuanto a procesos, valores y concepciones manejados por el alumnado y el profesorado de los centros estudiados, y en la relación entre el trabajo con tecnologías digitales y el desarrollo de una educación transformadora. Objetivo al que debe dirigirse la escuela.

\subsection{Dimensión organizativa: un nuevo sistema relacional para la escuela digital}

Vivimos en una sociedad digital en la que las tecnologías no son solo elementos imprescindibles en nuestras vidas cotidianas, sino que gozan de una amplia aceptación, legitimidad y prestigio como elementos de avance y mejora social, sumiéndose en lo que Cancela (2019) ha venido a denominar como "sueño tecnológico". Ante esta somnolencia ciudadana, seducida por el potencial onírico de las tecnologías digitales, argumenta San Martín (2009, p. 217) que "la organización escolar, aunque se resista, tampoco escapará a la tendencia de ser tecnológicamente modificada".

Este fenómeno es claramente visible en los centros escolares estudiados, que utilizan una gran variedad de dispositivos (ordenador, iPad, Chromebook, PDI, robots, drones, Smart TV, etc.) y plataformas y aplicaciones digitales como elementos cotidianos del centro escolar, tal y como plantean los Coordinadores TIC entrevistados:

Las TIC están incluidas en el centro, todo se trabaja desde las TIC. En las aulas, en administración, el trabajo interno del claustro de profesores, la comunicación con las familias. (CCV_ECTIC, I. 66-67).

Para dar cabida a estas herramientas la escuela y sus procesos se transforman. Las tecnologías digitales, siguiendo a Castells (2001), no son meras herramientas de comunicación o trabajo, sino que configuran nuevas geografías en las que mutan tanto las formas como los valores, principios y lógicas que las sustentan:

Planificamos un plan de implantación de la tecnología desde infantil hasta bachillerato [...] y ahí intentamos no solo trabajar el ámbito técnico o competente del uso de las herramientas sino la conciencia y la identidad de las personas con el uso de internet. (CCM_ETIC, I.100-115). 
En el contexto escolar explorar las nuevas geografías digitales comporta cambios no solo en la esfera didáctica, como se abordará posteriormente, sino también en la organizativa. Fruto de ello, los espacios escolares se reconfiguran, como reconocía uno de los docentes:

Como el diseño arquitectónico se decidió cuando se empezó con el proyecto, apostamos por tener pasillos casi más amplios que las aulas, ya que se utilizan como zonas de estar para que el alumnado de las diferentes clases se pueda encontrar (CCM_ET, I. 68-77).

Y, en ocasiones, incluso el espacio se organiza tomando las tecnologías como elemento articulador, en base a modelos como el del Aula del Futuro:

Con espacios flexibles, abierta, con mogollón de materiales manipulativos, vivenciales y también tecnológicos [...], tendremos una pared pintada de verde donde te vale para hacer Chromas y también para visualizar [...]. También tendrás para poner absolutamente lo que quieras, te pasas la imagen del iPad y lo podrás compartir entre varios. Todos esos espacios. Hay gafas de realidad virtual ahí para que te las puedas poner y puedas ver directamente. (CPV_ECTIC, I. 603-633).

Esta reconfiguración organizativa no se limita a los espacios, sino que también atañe a la gestión de tareas, a los roles adoptados por los agentes escolares y a las relaciones que entre estos se establecen; reto que exige a la escuela un rediseño de sus dimensiones constitutivas.

En este sentido, el ámbito de la comunicación y las relaciones entre los agentes escolares experimenta también un profundo impacto. En relación al profesorado se alude a que las tecnologías propician el trabajo colaborativo y la formación docente, ofreciendo un amplio abanico de canales de comunicación instantánea, como expresaba uno de los docentes:

Tenemos un grupo de WhatsApp, [...] el servidor del centro que nos sirve muchísimo para poder contrastar, compartir trabajos, guardar trabajos, siempre tienen ahí ese fondo del material que necesitamos día a día. Luego tenemos nuestro propio correo g-mail corporativo y luego la plataforma Educamos. (CCV_ET6, I. 292-297).

Sin duda, las herramientas digitales facilitan la comunicación, aunque no resulta menos cierto que mayor cantidad no implica necesariamente mayor calidad. Además, la ubicuidad digital también abre vías al teletrabajo y sus consecuencias, como son la ruptura de cualquier barrera temporal o espacial, la ampliación de la jornada laboral y la vulneración del derecho a la desconexión: 
Una de las virtudes de tener el iPad es, por ejemplo, que si quieres hacer alguna tarea en casa, como tú tienes el correo del profesor y tu correo del cole, yo, por ejemplo, si se me olvida presentarlo, en casa el mismo día por la tarde le envío una foto. Y eso me parece muy correcto. (CPV_EA6, I. 336-339).

Fenómeno que afecta no solo a los docentes, sino también al alumnado, que manifiesta la pérdida de tiempo que le supone en ocasiones el trabajo digital:

Si no se cargaba bien la foto estabas dos horas, dos horas ahí intentando cargarla y perdías tiempo para hacer otras cosas. (CPV_EA6, I.134-135).

Con ello la introducción de tecnologías en la escuela configura nuevas coordenadas organizativas que definen el trabajo pedagógico que se realiza, lo que nos lleva a la siguiente dimensión.

\subsection{Dimensión didáctica: estrategias docentes digitales en la escuela innovadora}

Uno de los argumentos más frecuentes para fundamentar el trabajo digital en la escuela es el de la innovación educativa. Partiendo de esta visión, los centros escolares del estudio avanzan hacia nuevas formas de trabajo escolar que se presentan como renovaciones e innovaciones pedagógicas de gran potencial, y como necesarias para ofrecer una educación adaptada a las necesidades del alumnado y a los retos de la sociedad actual. Uno de los tutores manifestaba en la entrevista:

Yo creo que la actualización digital es, no buena, sino necesaria para estar al día y crear un vínculo con los alumnos. Si queremos transmitir contenidos y valores debemos estar cerca de sus puntos de interés y eso solo lo podemos conseguir si hablamos su idioma. Además, esta es la generación de la tecnología y si la obviamos estaremos muy lejos de acercarnos a ellos y conseguir lo que queremos conseguir con los chavales (CCV_ET6, I. 300-304).

En este escenario, se asocia la implementación de tecnologías digitales en el aula con la innovación educativa, ofreciendo una visión positiva, pero la innovación educativa digital va más allá de reproducir el libro de texto en el iPad. En los centros estudiados el profesorado vincula el trabajo con tecnologías a la innovación educativa y a la ampliación de los aprendizajes, como puede observarse en la siguiente cita:

El impacto es bestial porque se da toda la autonomía a la hora de trabajar. En cuanto al contenido, trabajas el contenido al límite al que el niño puede llegar, porque les vas dando todo lo que van necesitando en cada momento (CPV_ET6, I. 288-290). 
Visión que también es mantenida por el alumnado, que percibe un mayor aprendizaje con dispositivos digitales:

Parece que aprendemos un poco más con el iPad haciendo proyectos (CPV_EA5, I. 140).

Yo aprendo más con el ordenador, porque si no entiendes algo te da tiempo a buscarlo por internet. Y si estás con material, tienes que esperar a que todos acaben y preguntar y luego te tocará a ti (CCV_EA5, I. 250-252).

Por tanto, el impacto de la introducción de las estrategias digitales en los centros hace replantear a los claustros cómo afrontar el desarrollo e innovación de los contenidos educativos digitales. Es por ello que la evaluación de los procesos es una discusión permanente en algunos centros:

Es un proyecto grande que iniciamos con este grupo e intentamos tener dos enfoques uno que es el análisis sistémico (tener en cuenta la crítica del profesorado como la complejidad de las situaciones que vivimos todos como centro de nueva creación, que vamos a ver que en muchos casos las cosas se perciben de forma negativa porque puede percibirse como un trabajo adicional...) y luego desde intentar prototipar y evaluar que todos los pasos que vayamos dando se pongan a prueba. Para validar si lo que hacemos es correcto o no (CCM_ECTIC, I, 354-560).

No obstante, esta importancia de la evaluación no constituye una tendencia generalizada. Ya que, en algunos centros estudiados, pese a que las visiones manifestadas por alumnado y profesorado evidencian que la innovación educativa propiciada por las tecnologías se asume como un hecho incuestionable; se trata más bien de una apreciación no fundamentada en evidencias sólidas, porque no se realiza una evaluación global al respecto. Esta consideración apunta a dos fenómenos: la asociación entre tecnologías y metodologías docentes; y, la concepción subyacente a la innovación educativa.

Por un lado, en cuanto a la relación entre tecnologías y metodología, en los centros estudiados, los agentes escolares frecuentemente "confunden" ambos elementos y asocian el impacto producido por una u otras de manera equivalente, contemplándolas como partes de un mismo plan de acción. Esto provoca que las "partes" se confundan y se obvie que "el todo" es mucho más que su simple suma. Este fenómeno responde a que en los centros la introducción de tecnologías en las aulas suma sinergias con la implementación de cambios metodológicos relacionados con las denominadas "metodologías activas" como el Aprendizaje Basado en Proyectos, el Aprendizaje por Servicio, el aprendizaje cooperativo, etc., como una de las coordinadoras indicaba:

Como base de innovación es: no tenemos libro de texto, la metodología que utilizamos que es sobre todo la metodología cooperativa, con todas sus técnicas, y luego todos los proyectos que incluimos en el centro (CPV_ECC, I. 75-77). 
Estos centros abogan por adaptarse a la realidad y a las demandas del contexto ofreciendo una renovación pedagógica que frecuentemente combina la introducción de tecnologías con la aplicación de estrategias docentes activas como base para ofrecer un proyecto educativo innovador. De forma que, en muchas ocasiones, las apreciaciones que se realizan respecto al impacto de las tecnologías digitales, en realidad no valoran este aspecto, sino los cambios propiciados por la metodología.

Recibimos mucha formación sobre metodologías activas (...) pero lo importante es la metodología activa, el aprendizaje por proyectos. La tecnología está ahí para ayudarnos en el aprendizaje de las metodologías activas. (CCM_ECC, I. 200-219).

Además, la asociación establecida entre innovación y trabajo digital parte de una visión restringida y utilitarista de la innovación, supeditada a las demandas del contexto social y del mercado de trabajo, lejana a lo expuesto por Gimeno (2011), respecto a que la verdadera innovación comporta no solo una renovación de los aspectos formales, sino una transformación y optimización de la realidad del aula.

Esto implica que, revestidas con la apariencia de la innovación y el progreso, se perpetúen metodologías, prácticas y concepciones del aprendizaje mecánicas y reproductoras, que difieren escasamente de las tradicionales, como expone un alumno:

Cuando tenemos clase de matemáticas, nos metemos al libro digital, buscamos la página, el profesor lo pone en la pantalla digital y ahí nos va explicando todo. Después, para poner los ejercicios, nos los asigna a nosotros en un apartado que nos pone tareas asignadas, pues ahí le damos y nos aparecen todos los deberes que tenemos que hacer. Puede ser que te digan que lo hagas en libreta o... (CCV_EA5, I. 311-315).

No solo se perpetúan las viejas prácticas sino que también se legitima el "halo tecnófilo" imperante en el panorama educativo actual, la visión de las tecnologías como elementos para la innovación y la mejora, como evidencia otra alumna:

Ahora como todos tenemos el ordenador, eso no tiene nada que ver, pero antiguamente, cuando íbamos a cuarto que no teníamos dispositivo, podíamos hacerlo o digital y meterlo en un pendrive, o escrito. Si lo hacías digital tenías más nota porque quedaba más aseado, más bonito, pues todo se entendía mejor a que si lo hacías en una cartulina que ponías tres líneas y una foto o un dibujo, que quedaba más cutre y siempre te iba a salir mejor haciéndolo en digital (CCV_EA5, I. 425-431). 
Orientación que también manifiestan los docentes:

Los iPads en concreto nos ofrecen aplicaciones que permiten generar un producto espectacular donde el alumno pueda contar todo lo que ha aprendido de una forma muy sencilla. Puedes generar un producto muy atractivo. Que no es la típica forma de contarlo en un mural (CPV_ECTIC, I. 101-104).

Este tipo de valoraciones y prácticas propicia que el alumnado, y también de manera menos consciente el profesorado, no priorice los objetivos de aprendizaje, sino los de "rendimiento", que pueden tener valor de cambio y no de uso, como expone Santos (2014). En cierta forma, el trabajo digital permite, cuando no fomenta, un tipo de aprendizaje en el que prima el trabajo fácil, la inmediatez, en detrimento de la constancia, la capacidad de esfuerzo y la perseverancia:

Yo busco una palabra en el diccionario y tengo que estar buscando la letra, y dentro de esa letra la página, la palabra... Y con el iPad busco la palabra y me sale (CPV_EA6, I. 543-544).

El alumnado entrevistado reconoce abiertamente este fenómeno, y argumenta su preferencia por el trabajo digital aludiendo a la inmediatez, en línea con la sociedad digital actual, caracterizada por la aceleración y la volatilidad:

Porque es más fácil encontrar como la página y tú ves todo lo que están viendo los profes. En cambio, en el libro, tienes que ver todo (CCV_EA5, I. 271-272).

Para mí es más fácil el ordenador, porque en hojas te cansas mucho escribiendo. Y en cambio, en el portátil no (CCV_EA5, I. 276-277).

Prefiero los Materiales Digitales, porque si no cada ejercicio tenías que copiar todas las líneas y digitales es más fácil (CCV_EA6, I.222-223).

Con ello el proceso educativo pierde su carácter globalizador e integral y se instrumentaliza, lo que nos lleva a cuestionarnos el impacto de este enfoque y sus prácticas en la formación de la ciudadanía.

\subsection{Dimensión social y ciudadana}

Ofrecer una educación capaz de avanzar hacia una sociedad más cohesionada, justa e igualitaria, constituye uno de los principales desafíos de la escuela actual. En un panorama altamente digitalizado, la introducción de tecnologías en los procesos educativos puede contribuir a alcanzar tales objetivos propiciando nuevos aprendizajes que velen por un desarrollo social, ético y sostenible, favoreciendo la igualdad de oportunidades y atendiendo a 
la gran diversidad existente, tanto en relación a las capacidades, como a la procedencia geográfica, étnica, de género, etc., con el objetivo de crear una escuela y una sociedad inclusivas.

Desde esta perspectiva social y ciudadana, uno de los grandes retos que se plantean las escuelas analizadas (y que constituye una gran preocupación a nivel nacional e internacional) es favorecer la igualdad de oportunidades de aprendizaje, avanzando hacia la inclusión. Las tecnologías digitales no deben convertirse en un elemento más de discriminación, sino que pueden abrir nuevas posibilidades para adaptar y personalizar los ritmos y procesos de aprendizaje, convirtiéndose en un eslabón clave para la integración, desarrollo y participación del alumnado como ciudadanos activos de la sociedad, como reflejan los entrevistados:

Tienes que saber dónde sacar mejor partido a uno de tus alumnos [...] prepararte las sesiones de diferentes maneras, incluso la misma explicación que vayas a hacer, hacerla de diferentes formas para saber que vas a llegar a todos los niños (CPM_ET, I. 80-85).

Para poder llegar a todos digitalmente, siguiendo a Cabero y Córdoba (2009), se deben dar una serie de medidas para favorecer la inclusión digital, y ello pasa por facilitar la presencia y el acceso a las tecnologías; revisar sus características de adaptabilidad y accesibilidad, y la alfabetización digital. Como afirma uno de los docentes entrevistados:

De esta forma puedes trabajar de manera más individualizada, variar los recursos con tu iPad y presentarlo a cada niño con diferente contenido (CPM_ET, I. 91-93).

En ambas comunidades autónomas el profesorado utiliza las tecnologías como herramienta para atender a la diversidad, aprovechando su potencial inclusivo, como refleja uno de los docentes al afirmar que:

Este uso de las herramientas digitales en realidad lo que hace es que minimiza las diferencias entre ellos (CPV_ET6, I. 144-148).

A este posicionamiento se une, de forma paralela, el interés por atender a la perspectiva de género. Ambas cuestiones son consideradas como elementos fundamentales para dar respuesta a una escuela comprometida con la equidad y la justicia social.

En clase trabajamos casi siempre por equipos. Los equipos los hacen los profesores pensando en dos cosas: una que seamos el mismo número de niños y de niñas; y la otra, que cada uno tenemos una función en el equipo (CPM_EA, I. 57-62). 
Además de estas medidas específicas, el profesorado intenta abordar la perspectiva de género, mediante su inclusión de forma transversal en los distintos procesos y actividades desarrollados, y en la propia vida cotidiana del aula, como evidencia una de las coordinadoras:

El género se trabaja de forma natural durante el día a día [...] sobre todo en el vocabulario [...], y para ello se han hecho actividades concretas en dinámicas de grupo, pero se intenta generar de forma natural (CPV_ET5, l. 147-153).

La perspectiva de género pasa a formar parte no solo del currículum explícito, sino especialmente del oculto, del día a día, de las formas y modelos de interacción y relación que se tejen entre los agentes escolares. No obstante, al contrario que en el caso de la atención a la diversidad funcional, en relación a la perspectiva de género no se han podido constatar evidencias, respecto a la contribución de las tecnologías digitales hacia tal finalidad en ninguno de los centros estudiados. En este sentido, se aprecia una ausencia respecto al potencial que ofrecen las tecnologías, el cual la escuela, ante la complejidad y magnitud de aspectos a los que atender, no siempre es capaz de aprovechar. Del mismo modo, también cabría destacar otras ausencias como el papel de las tecnologías para promover la sostenibilidad o la participación extensa de la comunidad educativa.

Respecto a la sostenibilidad, desde las escuelas analizadas en ambas comunidades autónomas, es un concepto poco trabajado, sobre el que se han podido extraer escasas evidencias. Tan solo en una de las escuelas el coordinador digital apuntó que:

Uno de los principios del centro es usar, en la medida de lo posible, software libre y reutilizar equipos. Este principio es complejo porque al final la reutilización de equipos supone un mayor coste de personal que los pongan en condiciones (CCM_ECTIC, I. 180-190).

Y en otro de los centros se muestra una preocupación por la sostenibilidad medioambiental relacionada con la disminución del uso de papel, favorecida por la sustitución de los elementos impresos por digitales, como manifiesta una alumna:

Tenemos menos peso en la mochila y menos papeleo. Y eso mola (CPV_EA6, I. 269).

Pese a estas referencias, las acciones son puntuales, por lo que resultan de escasa efectividad. Además, no responden a una perspectiva holística sobre la sostenibilidad, en línea con los planteamientos de la Agenda del Desarrollo Sostenible 2020, sino que se focalizan exclusivamente en la esfera medioambiental. Y tampoco en este ámbito se produce un impacto 
profundo, puesto que pese a que a priori las acciones desarrolladas pueden catalogarse como medidas sostenibles, no debemos obviar otras problemáticas relacionadas con el impacto de la transformación digital sobre el medio ambiente, por ejemplo, la huella de carbono, la obsolescencia digital, etc.

Finalmente, tampoco en el ámbito de la participación se ha podido constatar una mejora derivada de la digitalización. Anteriormente aludíamos a los cambios en la relación entre docentes y discentes, destacando que cantidad y calidad no siempre vienen de la mano. Aspecto que también es aplicable a la relación entre familia y escuela. En los centros estudiados se utiliza una gran variedad de herramientas digitales para facilitar la participación de las familias, con el objetivo de motivar su implicación al proporcionar una comunicación más fluida y rápida, como explican los docentes:

Tenemos la web del cole [...] utilizamos Telegram, utilizamos grupos de WhatsApp con las familias, a parte de ITACA, ITACA-familias, el Drive, Classroom, sí, tenemos muchas vías, muchos canales de comunicación. (CPV_EED, I. 223-226).

No obstante, pese a esta voluntad, lo cierto es que la relación digital mediatizada por las plataformas está muy lejos de propiciar una verdadera participación en sentido extenso, necesaria para el desarrollo de una educación democrática encaminada al ejercicio de una ciudadanía activa. Sino que aboga por una "participación de mínimos" incluso asentada en una perspectiva clientelar.

Estas ausencias detectadas visibilizan que, al igual que sucedía con el aspecto de la innovación, en torno al trabajo digital se ponen en circulación discursos y valores asumidos a nivel social pero escasamente cuestionados, sobre los que resulta necesario trabajar y reflexionar.

\section{Discusión y conclusiones}

El contexto actual, marcado por la globalización, la incertidumbre y la transformación digital, plantea numerosos retos a los profesionales del sector educativo (Bauman, 2011). En un mundo complejo, la educación adquiere una importancia fundamental, no solo para propiciar el desarrollo óptimo de los individuos sino también para "ayudar a comprender el mundo y a comprender al otro para así comprenderse a sí mismo" (Delors, 1996, p.31); fomentando la creación de una ciudadanía activa, comprometida con la transformación y optimización social, y con la superación de las numerosas 
desigualdades que persisten en la sociedad actual. Desafío que exige una educación sensible al contexto, enraizada en la realidad y que se prolongue durante toda la vida.

Para conseguirlo, la escuela y sus profesionales tratan de adaptarse a las necesidades y demandas del contexto social. De esta forma, en la actual sociedad digital, también la escuela se digitaliza progresivamente. Fenómeno que se ha podido constatar durante la investigación realizada, que refleja el innegable impacto de las tecnologías digitales en todos los ámbitos de la vida escolar, tanto en la esfera organizativa como en la didáctica.

En relación a la dimensión organizativa, los principales cambios observados atañen a la reconfiguración de espacios para posibilitar el trabajo digital, a la gestión de tareas realizadas a través de nuevos formatos digitales, y a la transformación del sistema de relaciones entre alumnado, profesorado y familias. Lo que repercute en una mutación profunda de las distintas dimensiones constitutivas de la organización escolar, en proceso de transformación digital.

Por lo que respecta a la dimensión didáctica, los centros estudiados caminan hacia la implementación de estrategias didácticas digitales. Los equipos docentes avalan y potencian tal fenómeno, manifestando una visión prácticamente unánime respecto al potencial innovador de las tecnologías, destacando la posibilidad de crear material propio y de individualizar los procesos de enseñanza-aprendizaje, adaptándolos al contexto concreto. No obstante, la investigación también visibiliza ciertas debilidades o carencias del trabajo digital, como el reduccionismo en cuanto a la innovación o la facilidad y la inmediatez que provocan que el proceso de enseñanza-aprendizaje no siempre ponga el foco en los objetivos y contenidos curriculares. Situaciones que desencadenan fenómenos de mayor envergadura, y que incluso provocan una reorientación de los fines de la educación. Puesto que en una época caracterizada por la inmediatez impuesta por el acelerado desarrollo tecnológico, el progreso se mide frecuentemente en términos reduccionistas y técnicos, vinculados al crecimiento económico. Con ello, el proceso educativo en ocasiones se supedita al servicio y a los mandatos de los valores y la lógica que priman en el contexto social, mediatizado por el capitalismo digital (Schiller, 1999), obviando la importancia de la dimensión social y ciudadana para avanzar hacia la optimización de la sociedad.

Sin embargo, el desarrollo tecnológico no puede realizarse a expensas del desarrollo social. Y la escuela debe velar y contribuir activamente a que la introducción de tecnologías digitales no se convierta en un elemento de discriminación; sino que, al contrario, actúe como articulador de puntos de encuentro con la diversidad y la alteridad, propiciando experiencias de 
aprendizaje para la vida basadas en la interdisciplinariedad, en la configuración de redes colaborativas, en el aprendizaje experiencial y en la realidad vital del alumnado.

A pesar de estos deseos y de las potencialidades de las tecnologías, la inmersión en los centros seleccionados ha puesto de manifiesto que aún queda mucho camino por seguir recorriendo, tal y como el propio profesorado reconoce:

Mucho nos queda por aprender de cómo crear una escuela tecnológica que dé respuesta a todas las cuestiones que nos planteamos y analizamos en los claustros y equipos directivos (CCM_ETIC, I. 200-208).

Las tecnologías no parecen haber introducido en el sistema educativo los cambios necesarios que permitan el abordaje profundo y comprometido de cuestiones de gran calado. Esta realidad limita las posibilidades de aplicación práctica y de cambio en la escuela (Sánchez-Antolín y Blanco, 2016). Para conseguir este viraje, esta atención a lo que realmente importa para el desarrollo integral de la ciudadanía, es necesaria la articulación del currículum, la organización de tiempos y espacios, la selección de los materiales didácticos y realizar modificaciones en los sistemas de evaluación, además de fomentar la formación del profesorado y la participación de la comunidad en el proceso de enseñanza-aprendizaje.

En conclusión, uno de los principales retos de la escuela y de la investigación continúa siendo reflexionar sobre cómo introducir las tecnologías digitales con finalidad transformadora y optimizadora, dirigidas a fomentar el desarrollo social y ciudadano del alumnado, para así avanzar hacia una sociedad más cohesionada, democrática, abierta, sostenible y comprometida. Aspecto que vuelve a poner de manifiesto lo apuntado al inicio del presente trabajo, respecto a la importancia de dotar de herramientas al alumnado para que tenga la capacidad de interpretarse e interpretar el mundo que les rodea, para así poder transformarlo, situación que también reflejaban los propios docentes entrevistados:

Presentarles el nuevo mundo que se les abre con las nuevas tecnologías no es fácil. (CPM_ET, I. 108-115).

Este ambicioso reto no depende exclusivamente de los centros escolares y sus claustros, sino que exige una estrecha colaboración con la comunidad educativa. Por lo que, de nuevo, destaca la importancia de avanzar hacia una participación democrática extensa de todos los agentes escolares y, sobre todo, de abrir la escuela a la vida y a la realidad. 


\title{
Agradecimientos
}

Proyecto emergente Análisis de las estrategias docentes ante la digitalización de los contenidos del currículum de Educación Infantil y Primaria (GV/2018/074) financiado por la Conselleria de Educación, Investigación, Cultura y Deporte.

\author{
Proyecto de tesis doctoral con referencia FPU16/04009 financiado \\ por el Ministerio de Ciencia, Innovación y Universidades del Gobierno Español.
}

\section{Referencias}

Amores, A.J. y De Casas, P. (2019). El uso de las TIC como herramienta de motivación para alumnos de enseñanza secundaria obligatoria. Estudio de caso español. HAMUT'AY, 6(3), 37-49.

Area, M. y Rodríguez, J. (2017). De los libros de texto a los materiales didácticos digitales. RELATEC, 16(2), 9-12.

Bauman, Z. (2011). Los retos de la educación en la modernidad líquida. Barcelona: Gedisa.

Buxarrais, M.R. y Ovide, E. (2011). El impacto de las nuevas tecnologías en la educación en valores del siglo XXI. Sinéctica, 37, 1-14.

Cabero, J. y Córdoba, M. (2009). Inclusión educativa: inclusión digital. REI, 2(1), 61-77.

Cabero, J. y Valencia, R. (2019). TIC para la inclusión: una mirada desde Latinoamérica. Aula Abierta, 48(2), 139-146.

CAM (2010). Orden 1275/2010, de 8 de marzo, de la Consejería de Educación, por la que se implanta el proyecto de institutos de innovación tecnológica en la Comunidad de Madrid. Boletín Oficial de la Comunidad de Madrid, 65, 51-60. Accesible en https://bit.ly/33pwKu5

Cancela, E. (2019). Despertar del sueño tecnológico. Madrid: Akal.

Castells, M. (2001). La galaxia internet. Madrid: Areté.

Castro, M., Marín, D. y Sáiz, H. (2019). Competencia digital e inclusión educativa. Visiones de profesorado, alumnado y familias. RED, 61, 1-37. https://doi.org/10.6018/red/61/06

Colás, M.P., De Pablos, J. y Ballesta, J. (2018). Incidencia de las TIC en la enseñanza en el sistema educativo español: una revisión de la investigación. RED, 56, 1-23.

Conde, S., Ávila, J.A., Núñez, L. y Mirabent, M.D. (2015). Opinión del profesorado y alumnado sobre la implantación, uso y resultados de las TIC en Educación Primaria: evaluación de un Centro. REICE, 13(3), 57-75.

Delors, J. (1996). La Educación encierra un tesoro. Unesco. 
Fernández, F.J., Fernández, M.J. y Rodríguez, J.M. (2018). El proceso de integración y uso pedagógico de las TIC en los centros educativos madrileños. Educación XXI, 21(2), 395-416. https://doi.org/10.5944/educXX1.17907

Gallardo, I.M.; San Nicolás, A.B. \& Cores, A. (2019). Visiones del profesorado de primaria sobre materiales didácticos digitales. Campus Virtuales, 8(2), 47-62.

Gimeno, J. (Coord.) (2010). Saberes e incertidumbres sobre el currículum. Madrid: Morata.

Loveless, A. y Williamson, B. (2017). Nuevas identidades de aprendizaje en la era digital. Madrid: Narcea.

Martín, S.G. y Mayo, I.C. (2019). Uso de tecnologías y rendimiento académico en estudiantes adolescentes. Comunicar, 59, 73-81.

Martínez-Serrano, M.C. (2019). Percepción de la Integración y uso de las Tecnologías de la Información y la Comunicación (TIC). Estudio de Profesores y Estudiantes de Educación Primaria. Información tecnológica, 30(1), 237-246.

Pardo, M.I. San Martín, Á. y Cuervo, E. (2019). La performatividad docente en el entorno digital de los centros escolares: redefinición del trabajo didáctico. ReiDoCrea, 8(2), 6-18.

Pardo, M.I. y San Martín, Á. (2020). Tecnologías y cultura organizativa en los centros escolares. ¿La uberización de las relaciones laborales?, PíxelBit, 58, 161-179. https://doi. org/10.12795/pixelbit.72767

Patton, M.Q. (2002). Qualitative evaluation and research methods (3 rd ed). Newbury Park. CA: Sage.

Peirats, J., Gallardo, I.M. y San Martín, Á. (2015). Implementación de dispositivos digitales en las aulas valencianas de primaria. En J. de Pablos Pons (coord.), Los centros educativos ante el desafío de las tecnologías digitales (pp. 199-220). Madrid: La Muralla.

Rego-Agraso, L. y Marín, D. (2019). Las visiones del alumnado sobre los Materiales Didácticos Digitales en España. Educar em revista, 35(77), 79-94.

Saladino, M., Marín, D. y San Martín, Á. (2019). Aprendizaje mediado por tecnología en alumnado con tea. Una revisión bibliográfica. Etic@net, 1, 1-25.

San Martín, Á. (2009). La escuela enredada. Formas de participación escolar en la sociedad de la información. Barcelona: Gedisa.

San Martín, Á., Peirats, J. y Gallardo, I. M. (2014). Centros educativos inteligentes. Luces y sombras sobre las políticas de transferencia de tecnología y las prácticas docentes. Profesorado. Revista de currículum y formación del profesorado, 18(3), 63-79.

Sánchez, N., Ortega, O. y Vall-Llovera, M. (2012). Romper la brecha digital de género. Factores implicados en la opción por una carrera tecnológica. Athenea Digital, 12(3), 115-128.

Sánchez-Antolín, P. y Blanco, M.M. (2016). La política educativa TIC de la Comunidad de Madrid (España): la perspectiva del profesorado. RELATEC, 15(1), 45-58. https:// doi.org/10.17398/1695288X.15.1.45

Santos, M.Á. (2014). El arca de Noé: la escuela salva del diluvio. Guadalajara: ITESO.

Schiller, D. (1999). Digital Capitalism: Networking the Global Market System. Massachusetts: The MIT Press. 
Silva, I.T., Rodrigues, L.F. y Sampayo, M.M. (2020). The bad and the ugly: a systematic review of technology's negative impacts' mentions in literature from 2005 to 2020. 6th International Conference on Higher Education Advances (HEAd'20). Universitat Politecnica de València, València. https://doi.org//10.4995/HEAd20.2020.11152

Vidal, M.I., Vega, A. y López, S. (2019). Uso de materiales didácticos digitales en las aulas de Primaria. Campus Virtuales, 8(2), 103-119. 